\title{
Endoscopic Endonasal Approach of Orbital Medial Wall Reconstruction using Septal Cartilage: A Rare Surgical Case Report
}

\author{
${ }^{1} \mathrm{MK}$ Rajasekar, ${ }^{2} \mathrm{M}$ Vivek, ${ }^{3} \mathrm{~V}$ Narendrakumar
}

\begin{abstract}
Orbital fractures can cause facial disfigurement and disturbs vision. Medial orbital wall fractures are diagnosed with frequent use of computed tomography (CT) scans in the diagnosis of orbital trauma. Orbital reconstruction plays a challenging role for the surgeons as it comes in a multitude of different forms. We report a case of 45-year male with a history of assault presented with diminution of vision, restricted abduction and diplopia of the left eye. Computed tomography scan showed fracture of the left medial orbital wall fracture and the patient was treated by endoscopic endonasal approach of medial orbital wall reconstruction using septal cartilage.
\end{abstract}

Keywords: Blow out fracture, Endoscopic, Lamina papyracea, Medial orbital wall.

How to cite this article: Rajasekar MK, Vivek M, Narendrakumar V. Endoscopic Endonasal Approach of Orbital Medial Wall Reconstruction using Septal Cartilage: A Rare Surgical Case Report. Clin Rhinol An Int J 2015;8(1):30-33.

Source of support: Nil

Conflict of interest: None

\section{INTRODUCTION}

A medial orbital wall fracture mainly involves the lamina papyracea which is the thinnest bone of the orbit. The size of the defect may range in moderate to severe fractures from 13 to $20 \mathrm{~mm}$ in height and from 21 to 34 $\mathrm{mm}$ in length. ${ }^{1}$ Incidence of medial orbital wall fracture is 0 to $10 \%$ of all blowout fractures. ${ }^{2}$ Most of the isolated medial orbital wall fractures need only conservative management. The presence of diplopia or limitation of eye movement is an indication for surgery for Blow out fractures of the medial orbital wall. ${ }^{3}$ Anatomic location of the displaced bone and the extent of the fracture play a main role in treatment outcome of Blow out fractures. ${ }^{4}$

${ }^{1}$ Professor, ${ }^{2,3}$ Formerly Postgraduate Student

1-3Department of ENT, Upgrade Institute of Otorhinolaryngology Madras Medical College, Chennai, Tamil Nadu, India

Corresponding Author: MK Rajasekar, Professor, Department of ENT, Upgrade Institute of Otorhinolaryngology, Rajiv Gandhi Government General Hospital, Park Town Chennai-3, Tamil Nadu, India, Phone: 09176054266, e-mail: stanvivek@gmail. com
Interpersonal violence is the most common cause of medial orbital wall fractures ${ }^{5}$ as in our case.

\section{CASE REPORT}

A 45-year-old male (Fig. 1) with history of assault presented with complaints of left nasal bleed, left periorbital ecchymosis, left diminution of vision and loss of consciousness at trauma ward. Computed tomography brain was taken showed no brain injury.

Then, he was referred to ophthalmology where ophthalmologist reported that his visual acquity on left side was 6/60 with conjunctiva congestion, mild restricted abduction and diplopia. Other parameters on left and right eye were normal. Computed tomography scan of orbit showed breach at medial wall of orbit -lamina papyracea (Figs 2A and 3). So, he was referred to ENT department for further management with cover of antibiotics and antiedema drugs.

We planned to take up for endoscopic endonasal surgery in two stages under general anesthesia. At first stage we found that the orbital fat (Fig. 4) was seen at the left side middle meatus (due to breach of lamina papyracea). Left side uncinectomy, middle meatal antrostomy was done; bulla and anterior ethmoidal air cells opened. Some of the protruded parts of the orbital fat were removed carefully and packed with surgicel. After a period of 1 week with antibiotics and antiedema drugs (edema settled down) 2nd stage surgery was done where we found the exact defect (approximately $1.5 \times 0.5 \mathrm{~cm}$ ) in the anteroinferior part of lamina papyracea (Fig. 2B). On the same side septoplasty was done and septal cartilage was taken

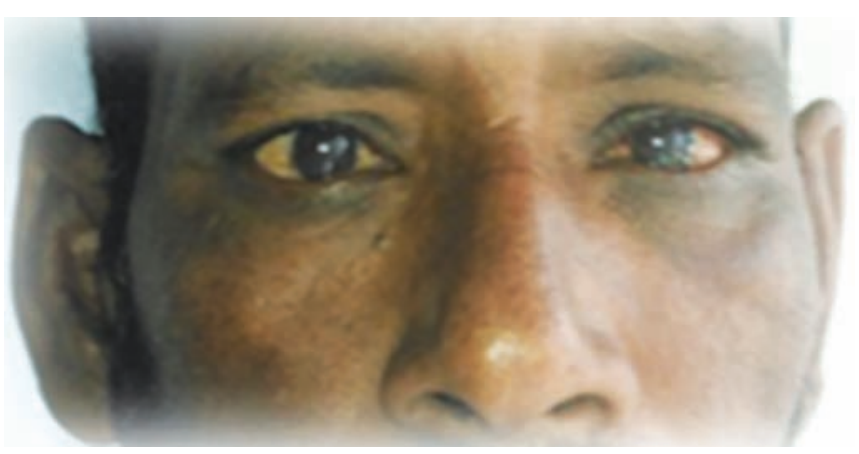

Fig. 1: Left eye ecchymosis 

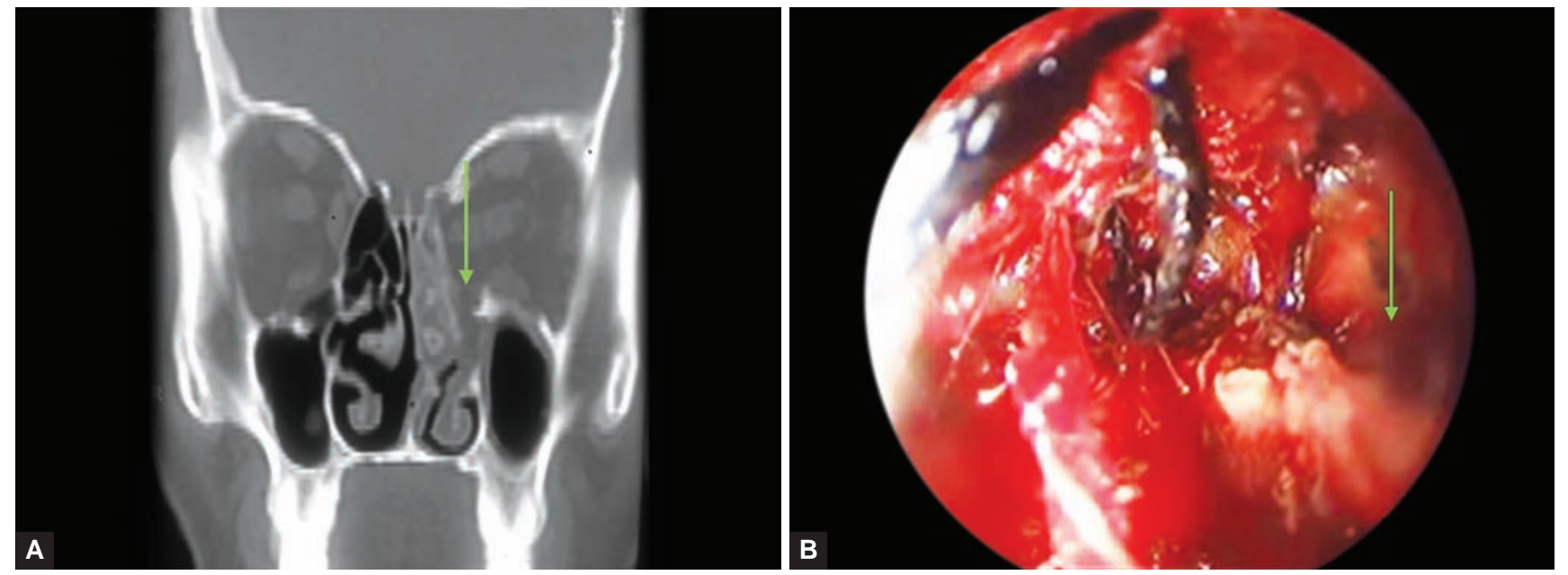

Figs 2A and B: (A) The defect in anteroinferior part of lamina at CT scan and (B) the defect in anteroinferior part of lamina intraoperatively

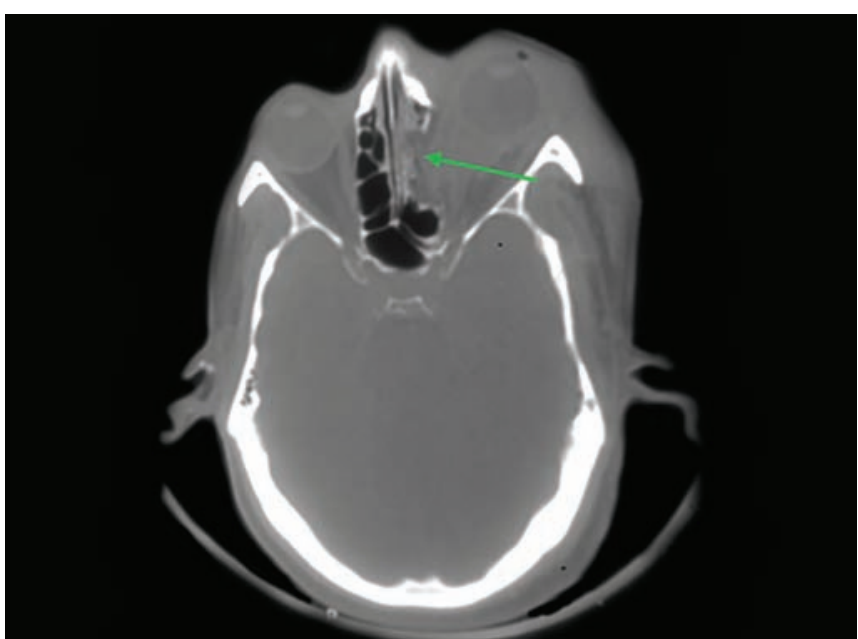

Fig. 3: The breach in left lamina papyracea

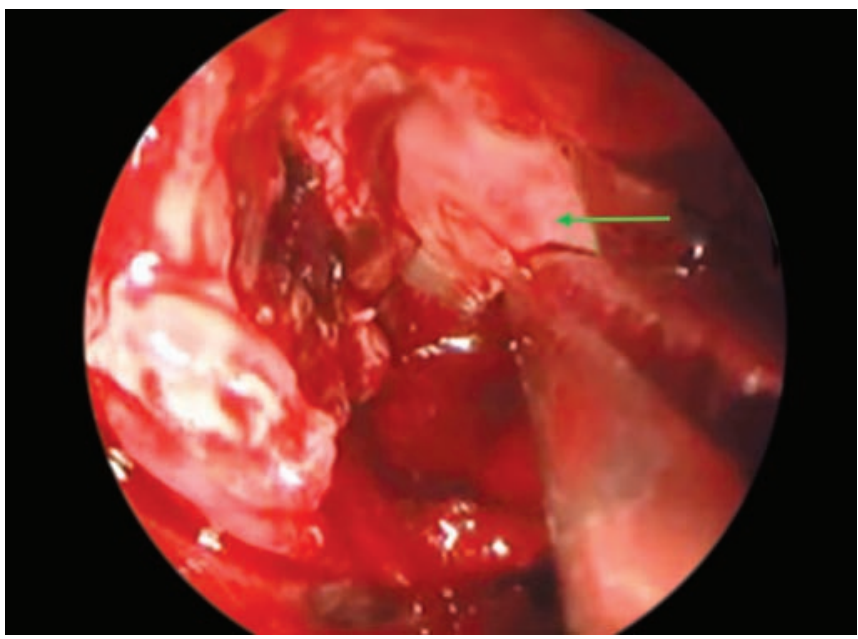

Fig. 5: The septal cartilage being placed at the defect in lamina papyracea

out and reshaped according to the defect in the medial wall of orbit. It was placed at the defect so that it covers the defect (Fig. 5) and supports the orbital contents from bulging in to the nasal cavity. Around the graft surgicel was packed and finally tissue glue applied. After 1 week,

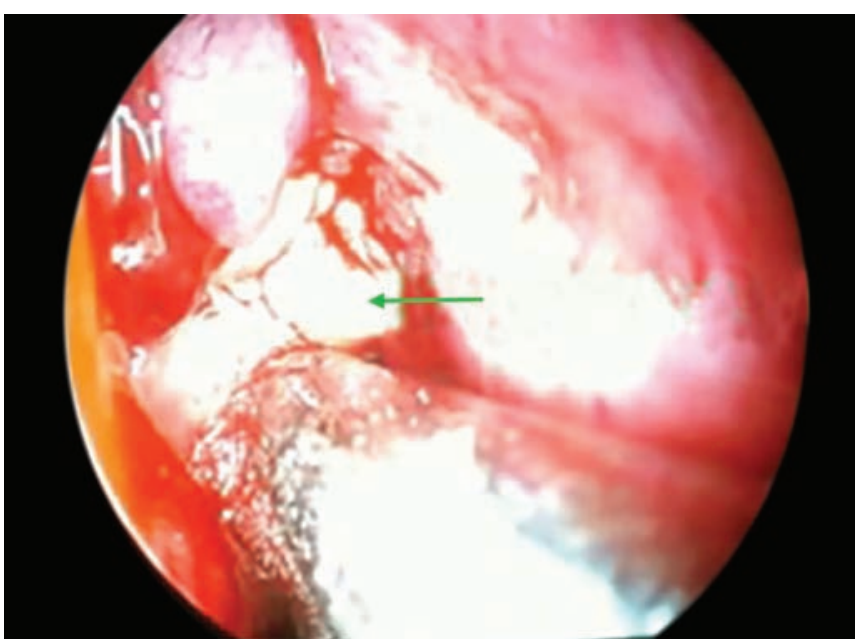

Fig. 4: Fat protruding at the left middle meatus

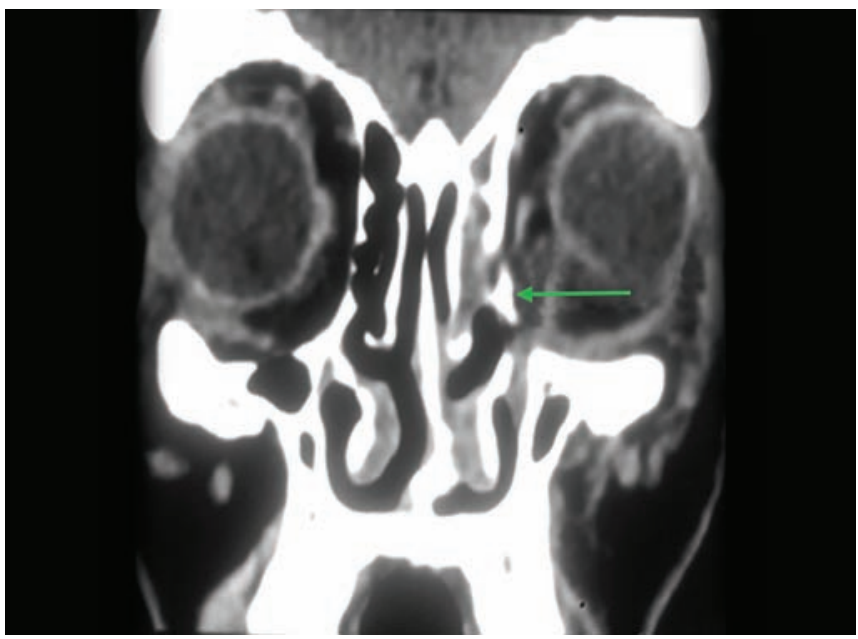

Fig. 6: Postoperative CT showing the septal graft in place at the defect

postoperative DNE and suction clearance was done. The surgical site healed well. Postoperative CT orbit (Fig. 6) was taken which showed the reconstructed medial wall of orbit. The left eye vision improved to 6/18 with normal (Fig. 7) eye movements and diplopia disappeared. 


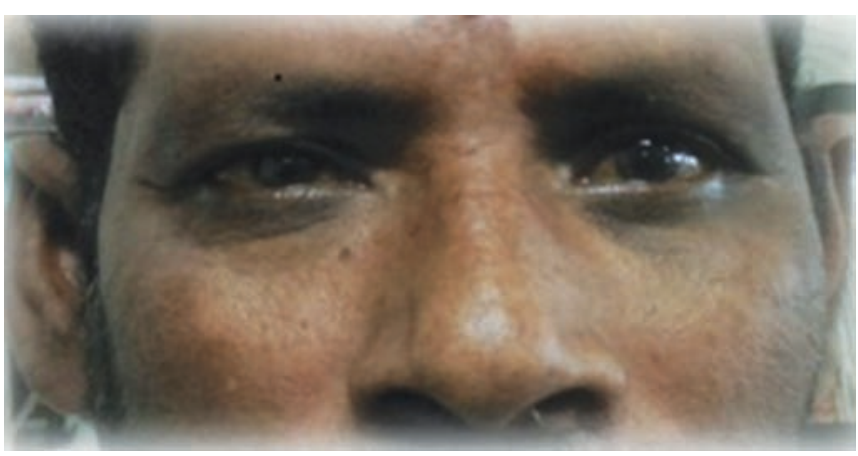

Fig. 7: Postoperative picture of the patient

\section{DISCUSSION}

Orbital fractures can cause functional disabilities and esthetic deformities. The medial wall is the second most commonly disrupted orbital wall ${ }^{6}$ which is formed by the frontal bone, the lacrimal bone, the lamina papyracea of the ethmoid, lesser wing of the sphenoid and anteriorly by the frontal process of the maxilla. The largest component and structural weakness of the medial wall is lamina papyracea bone. ${ }^{7}$ The occurrence of orbital fracture can be explained by two theories. They are: (1) increased fluid pressure can displace the eyeball posterior, (2) direct injury to the lower orbital wall can lead to inverted orbital floor. ${ }^{8}$

Jaquiéry et $\mathrm{al}^{9}$ classified orbital trauma into the following five classes:

- Class I: Small, isolated defects of the orbital floor or the medial orbital wall of approx. 1 to $2 \mathrm{~cm}^{2}$.

- Class II: Defects of the orbital floor and/or the medial orbital wall $>2 \mathrm{~cm}^{2}$. Bony structures of the medial wall of the infraorbital fissure are intact.

- Class III: Defects of the orbital floor and/or the medial orbital wall $>2 \mathrm{~cm}^{2}$, without bony structures of the infraorbital fissure.

- Class IV: Defects of the whole orbital floor and the medial wall to the infra orbital fissure.

- Category V: Same as IV, defect extending into the orbital roof.

Entrapment of the medial rectus muscle may occur if the medial orbital wall is fractured as it is within few millimeters of the lamina papyracea. ${ }^{10}$ Limitation of eye movement caused by entrapment of ocular muscles can be occurred due to fractured bony fragments, palsy of the oculomotor nerve, hematoma of ocular muscles, and herniation of orbital tissues.

Preferring a surgical approach to treat blowout fracture of the medial orbital wall has been a challenging task for surgeons. These approaches can be broadly divided into external and endonasal. De Visscher and van der Wa ${ }^{11}$ explained many approaches for exploration of medial orbital wall, which includes the conjunctival approach, subciliary incision, medial brow incision, medial canthal incision, incision above and below the medial canthal ligament, the open sky approach, and the ethmoidal approach.

Endonasal microscopic approach gave good results but with limitations like difficulties in visualizing all parts of the medial orbital wall. This limitation was overcome by preferring the endonasal endoscopic approach along with angled endoscopes which gives fantastic results by direct visualization of the fracture site with less potential for damaging the orbit and cosmetically good by avoiding a scar.

To prevent reherniation of orbital contents synthetic materials, like silastic sheet, porous polyethylene sheet, hydroxyapatite ceramic, and titanium mesh implants are used, but their long time presence can induce granulation tissue formation. Autogenous grafts harvested from ribs, calvaria, iliac bone, or the maxillary sinus wall is also used. ${ }^{12}$ In our case, we used autogenous nasal septal cartilage which gave an excellent result. It is advantageous because this graft is harvested adjacent to the surgical site.

\section{CONCLUSION}

An endoscopic endonasal approach of medial orbital wall reconstruction gives excellent visualization of the fracture site and offers safe removal of bony fragments, clean the traumatic changes of the ethmoid sinus and to reconstruct the blowout fracture. Another advantage of the endoscopic approach is that surgery can be performed under local anesthesia. This makes evaluation of ocular movements and the presence of diplopia possible during surgery. ${ }^{13}$ A successful medial orbital wall reconstruction depends on exact planning, precise surgical dissection, and proper selection of type, size, and contour of the reconstruction material. ${ }^{14}$

\section{ACKNOWLEDGMENT}

The authors are thankful to the Postgraduate Resident Dr Mohanbabu for efforts in collecting medical records of the patient.

\section{REFERENCES}

1. Burm JS, Chungch, Joon S. Pure orbital blowout fracture: new concepts and importance of medial orbital blowout fracture. Plastreconstr Surg 1999;103:1839-1849.

2. Chen CT, Chen YR, Tung TC, et al. Endoscopically assisted reconstruction of orbital wall fractures. Plast Reconstr Surg 1999;103:714-720.

3. Mathog RH. Management of orbital blow-out fractures. Otolaryngol Clin North Am 1991;24:79.

4. Manson PN. Facial injuries, in: McCarthy JG, Plastic Surgery. Philadelphia, PA: Saunders, 1990;2:1079-1080. 
5. Nolasco FP, Mathog RH. Medial orbital wall fractures: Classification and clinical profile. Otolaryngol Head Neck Surg 1995;112:549-556.

6. Lew D, Birbe J. Zygomatic complex fractures, in: Fonseca RJ, Marciani RD, Hendler BH, editor. Oral and Maxillofacial Surgery, 1st ed. Philadelphia, PA Saunders, 2000;3:185-186.

7. Pohlenz $\mathrm{P}$, et al. Medial orbital wall reconstruction with flexible Ethisorb patches. Clin Oral Invest 2013;17:511-516.

8. Lee CS, Yoon JS, Lee SY. Combined transconjunctival and transcaruncular approach for repair of large medial orbital wall fractures. Arch Ophthalmol 2009;127:291-296.

9. Jaquiéry C, et al. Reconstruction of orbital wall defects: critical review of 72 patients. Int J Oral Maxillofac Surg 2007;36: 193-199.
10. Naraghi M, Kashfi A. Endonasal endoscopic treatment of medial orbital wall fracture via rotational repositioning. Am J Otolaryngol 2002;23:312-315.

11. de Visscher JG, van der Wal KG. Medial orbital wall fracture with enophthalmos. J Craniomaxillofac Surg 1998;16:55-59.

12. van der Wal KG, de Visscher JG. Bone grafting for enophthalmos due to a medial wall fracture: Case report with 13-year follow-up. J Craniomaxillofac Surg 1999;27:177-179.

13. Hong-Ryul Jin, et al. Endonasal endoscopic reduction of blowout fractures of the medial orbital wall. J Oral Maxillofac Surg 2000;58:847-851.

14. Gabrielli MF, et al. Orbital wall reconstruction with titanium mesh: retrospective study of 24 patients. Craniomaxillofac Trauma Reconstr 2011 Sep;4(3):151-156. 